Schmerz 2014 $\cdot 28: 230-232$

DOI 10.1007/s00482-014-1435-1

Online publiziert: 6 . Juni 2014

(c) Deutsche Schmerzgesellschaft e.V.

Published by Springer-Verlag Berlin Heidelberg all rights reserved 2014

\author{
W. Jänig ${ }^{1}$ W. Häuser ${ }^{2}$ \\ ${ }^{1}$ Physiologisches Institut, Christian-Albrechts-Universität zu Kiel \\ ${ }^{2}$ Innere Medizin I, Klinikum Saarbrücken gGmbH
}

\title{
Viszerale Schmerzen
}

\section{Immer noch ein Stiefkind der Schmerzmedizin?}

Press (2005, 2008, 2013): 3/120 Artikeln (etwa 2\%)

kind der Schmerztherapie?" hatten wir vor 12 Jahren im Editorial zum Schwerpunkt „Viszerale Schmerzen“ bejaht [7]. Hat sich die Schmerzmedizin dem viszeralen Schmerz und die Viszeralmedizin der (symptomatischen) Schmerztherapie in den letzten 12 Jahren mehr angenommen? Die folgenden Ausführungen aus der Perspektive eines Grundlagenforschers und eines Klinikers sind als subjektive Auswahl der Autoren zu verstehen und erheben keinen Anspruch auf Vollständigkeit.

\section{Die Sicht des Grundlagenforschers}

Aus Sicht des Grundlagenforschers besteht weiter erheblicher Handlungsbedarf. Nach Durchsicht der relevanten Literatur (aus den Bereichen Wissenschaft, angewandte Wissenschaft und fortgeschrittene Lehre) der letzten 12 Jahre zum viszeralen Schmerz stellt sich Ernüchterung ein. Vor 12 Jahren gab Jänig in Der Schmerz eine Serie von 6 Arbeiten zum viszeralen Schmerz heraus [11]. Der Vergleich unseres damaligen Wissensstands mit dem heutigen lässt uns nachdenklich werden. Der viszerale Schmerz (Grundlagen und Klinik) ist in der Literatur im Vergleich zu anderen Schmerzen folgendermaßen repräsentiert:

- Encyclopedia of Pain [16]: 50/2678 Seiten (etwa 1,8\%)

- Refresher Courses International Association for the Study of Pain (IASP)
- Proceedings des 10., 11. und 12. Weltschmerzkongresses (IASP Press): 3/200 Artikeln (etwa 1,5\%)

- Bände über Schmerz in IASP Press (2002-2013): in 25 Bänden praktisch keine Abhandlung, in einem Band 93/565 Seiten $(<1 \%)$

- Wall \& Melzack's Textbook of Pain [14]: 90/1099 Seiten (Klinik: 6,8\%; Grundlagen: 1,4\%)

- Science of Pain [1]: 57/1017 Seiten $(5,6 \%)$

- Anfrage bei Medline 2002-2013: „visceral pain \& review“ im Vergleich zu „pain \& review“, Ergebnis: 729/56.415 Treffern (etwa 1,8\%)

Diese Zahlen drücken nach unserer Meinung schnörkellos aus, dass dieses klinisch wichtige Schmerzfeld in der Grundlagenforschung und in der klinischen Forschung unterrepräsentiert ist. Diese Unterrepräsentation korreliert

- mit einer sehr langsamen Entwicklung unserer Kenntnisse über die Mechanismen, die den viszeralen Schmerzen zugrunde liegen;

- mit der klinischen Erfahrung, dass sich die diagnostischen und therapeutischen Werkzeuge zur Behandlung der viszeralen Schmerzen in den letzten 12 Jahren nicht wesentlich geändert haben;

- mit den geringen Aktivitäten in der klinischen Forschung über die viszeralen Schmerzen und
- insbesondere mit der kleinen Zahl an Forschungslaboren, die sich mit den Mechanismen viszeraler Schmerzen experimentell beschäftigen.

Weltweit sind es weniger als 10 Labore, die sich hauptsächlich der Erforschung des viszeralen Schmerzes widmen - sie alle liegen in Australien, den USA und in England.

Diese besorgniserregende Situation hinsichtlich des viszeralen Schmerzes kann man auch inhaltlich untermauern [13]: Wir wissen im Grunde nach wie vor nicht, auf welche Weise Ereignisse bzw. adäquate Reize im Viszeralbereich, die zu Schmerzen führen, in der Aktivität viszeraler - im Wesentlichen spinaler - afferenter Neurone codiert werden. Wir können die Verarbeitung der afferenten viszeralen Aktivität durch die Neurone im Rückenmark, im Thalamus und im Kortex nicht beschreiben. Die Mechanismen der Sensibilisierung dieser peripheren und zentralen neuronalen Systeme bei akuten und chronischen Entzündungen im Viszeralbereich sind weitgehend unbekannt. Über die Mechanismen, die den Übertragungen viszeraler Schmerzen in die tiefen somatischen Gewebe, die Haut und in benachbarte viszerale Organe zugrunde liegen, können wir weitgehend nur spekulieren. Die Mechanismen der endogenen Kontrolle des zentralen viszeralen nozizeptiven Systems durch den Hirnstamm, welcher unter kortikaler Kontrolle steht, sind völlig unbekannt. Die zentralnervösen integrativen Prozesse der efferenten vegetativen und neuroendokrinen Syste- 
me in der Modulation viszeraler nozizeptiver afferenter Aktivität bleiben im Dunkeln.

Schlaglichtartig offenbar wird diese Situation dem Grundlagenforscher und Kliniker bei der Betrachtung von Patienten mit chronischen, potenziell generalisierenden Schmerzsyndromen, bei denen nach bisherigen Erkenntnissen die Nozizeptoren weder sensibilisiert noch durch Traumen geschädigt sind oder bei denen sensibilisierte Nozizeptoren keine offensichtliche oder nur eine untergeordnete Rolle in der Entstehung und Aufrechterhaltung der Schmerzen spielen. In diese Gruppe von Schmerzsyndromen gehören der Reizdarm („,irritable bowel syndrome“), die funktionelle Dyspepsie, der chronische Beckenschmerz („chronic pelvic pain“) oder der nichtkardiale Brustschmerz. Es wird vermutet, dass diese Schmerzen ihre Ursachen in zentralen Fehlregulationen der nozizeptiven Impulsübertragung, der affektiven Schmerzkomponente und der kognitiven Schmerzkomponente haben. Diese Schmerzen können nicht in die herkömmlichen Kategorien chronischer Entzündungsschmerzen oder neuropathischer Schmerzen eingeordnet werden, sondern bilden vermutlich eine eigene Kategorie chronischer Schmerzen [12]. Wir brauchen Grundlagenforschung, um dieses Konzept zu untermauern und $\mathrm{zu}$ beweisen.

\section{Wo besteht Handlungsbedarf aus Sicht des Grundlagenforschers}

Die Grundlagenforschung im Bereich des viszeralen Schmerzes an Tiermodellen (in vivo und in vitro) und am Menschen (gesunder Proband, Patient) ist technisch und intellektuell sehr anspruchsvoll. Diese Forschung ist aufwendig und kostet Zeit und Geld. Solch eine Forschung kann nur auf der Basis von Langzeitstrategien und -konzepten durchgeführt werden. Schnelle Ergebnisse sind nicht zu erwarten. Die Infrastrukturen an den Forschungsinstitutionen der Universitäten und den reinen Forschungsinstituten müssen diesem Sachverhalt Rechnung tragen und daran angepasst werden, will man den Geheimnissen viszeraler Schmerzen auf die
Spur kommen. Die Betonung liegt eindeutig auf der Förderung der Grundlagenforschung, was auch ganz besonders die Grundlagenforschung am Menschen als Modell in den klinischen Institutionen beinhaltet. Die Grundlagenforschung in den Forschungslaboren und in der Klinik muss erheblich besser integriert und gefördert werden. Sie kann nur auf der Basis einer Langzeitplanung funktionieren, die es jungen begabten Forschern ermöglicht, ihre Konzepte über längere Zeit (5-10 Jahre) zu verfolgen. Die Zielrichtung dieser Grundlagenforschung

- muss inhaltlich und methodisch breit angelegt sein und die Erforschung vegetativer und neuroendokriner Systeme einschließen und

- darf nicht primär an der Therapie viszeraler Schmerzen - d. h. am sog. Nutzen - und damit nicht an den klinischen Bedürfnissen ausgerichtet werden.

\section{Die Sicht des Klinikers}

Aus Sicht des Klinikers hat sich die Schmerzmedizin in den vergangenen Jahren mehr mit viszeralen Schmerzen und die Viszeralmedizin (Gastroenterologie, Gynäkologie, Kardiologie, Urologie, Viszeralchirurgie) mehr mit der symptomatischen Schmerztherapie befasst. Die Kampagne der International Association for the Study of Pain (IASP) von 2012/2013 war dem viszeralen Schmerz gewidmet [10]. Repräsentative Stichproben der deutschen Bevölkerung erlauben eine Abschätzung zur Häufigkeit von Bauchschmerzen in der allgemeinen Bevölkerung bei Kindern, Jugendlichen und Erwachsenen $[9,18]$. Für die peri- und postoperative Schmerztherapie bei großen viszeralchirurgischen Eingriffen wurden neue Techniken entwickelt [4]. In den USA wurden evidenzbasierte Leitlinien zum chronischen Unterbauchschmerz des Mannes und der interstitiellen Zystitis veröffentlicht [15]. In Deutschland wurde eine interdisziplinäre S2-Leitlinie zum chronischen Unterbauchschmerz der Frau erstellt [19]. In der europäischen Leitlinie zur Colitis ulcerosa und in den deutschen interdisziplinären S3-Leitlinien zu Morbus Crohn, Colitis ulcerosa, chronischer Pankreatitis sowie zum Reizdarmsyn- drom gibt es einen Abschnitt zur symptomatischen Schmerztherapie [3, 15]. Die deutsche interdisziplinäre S3-Leitlinie zur Neuromodulation enthält ein ausführliches Kapitel zur koronaren Herzerkrankung [6].

Der Anteil der Patientinnen mit chronischen Bauchschmerzen in gynäkologischen, internistischen, urologischen und viszeralchirurgischen Praxen und Kliniken kann nicht angegeben werden, da in den Diagnosestatistiken eine körperliche Krankheit, wie Endometriose, und nicht der chronische Schmerz codiert wird. Aus Deutschland liegen Daten zur Häufigkeit der Hauptdiagnose „viszeraler Schmerz“ aus schmerztherapeutischen Einrichtungen vor: Bei 3,5\% von 10.054 Patienten aus 19 schmerztherapeutischen Einrichtungen der Jahre 1999-2004 waren viszerale Schmerzsyndrome die Hauptdiagnose [5]. Am Universitätsklinikum Hamburg wurde die erste interdisziplinäre Sprechstunde für chronische Unterbauchschmerzen eingerichtet [2].

Für Symposien und Poster auf dem Deutschen Schmerzkongress gibt es inzwischen wenigstens die Kategorie „Varia“, in der Beiträge zu „Minderheitenthemen“ der Schmerzmedizin wie Gesichtsund Bauchschmerzen eingereicht werden können.

\section{Wo besteht Handlungsbedarf aus Sicht des Klinikers?}

Die Schmerzskizze im Deutschen Schmerzfragebogen ist ein geschlechtsloses Wesen ohne primäre und sekundäre Geschlechtsmerkmale. Ein Schmerz in der Brust, den Genitalien und im Damm kann nicht eingezeichnet werden. Ein $\mathrm{Zu}$ satzmodul wäre wünschenswert. Deutsche Leitlinien zur interstitiellen Zystitis (bzw. zum „painful bladder syndrome") und zum chronischen Unterbauchschmerz des Mannes fehlen. Das Kapitel zur Schmerztherapie der S3-Leitlinie zur chronischen Pankreatitis ist aus schmerztherapeutischer Sicht revisionsbedürftig [8]. Trotz der Häufigkeit chronischer Unterbauchschmerzen bei Frauen ist die Quantität und Qualität der Studien zur medikamentösen, physikalischen, psychotherapeutischen und multimodalen Therapie unzureichend. 
Wir hoffen, dass in einem Themenheft dieser Zeitschrift in 10 Jahren über weitere positive Entwicklungen in der Grundlagenforschung und Therapie viszeraler Schmerzen berichtet werden wird.

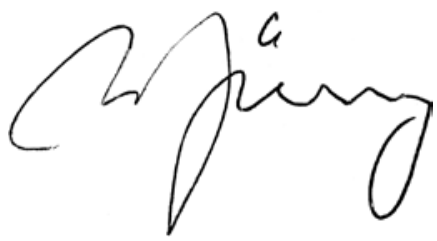

W. Jänig

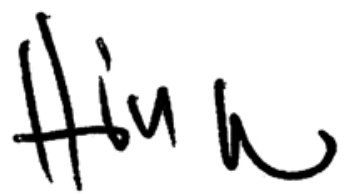

W. Häuser

\section{Korrespondenzadresse}

\section{PD Dr. W. Häuser}

Innere Medizin I, Klinikum Saarbrücken gGmbH Winterberg 1, 66119 Saarbrücken whaeuser@klinikum-saarbruecken.de

Interessenkonflikt. W. Häuser erhielt ein Beratungshonorar von Daiichi Sankyo und Vortragshonorare von Abbott, MSD Sharp \& Dohme und Pfizer in den letzten 3 Jahren. W. Jänig gibt an, dass kein Interessenkonflikt besteht.

\section{Literatur}

1. Basbaum Al, Bushnell MC (Hrsg) (2009) Science of pain. Academic Press, San Diego

2. Brühnahl A, Riegel B, Höink J et al (2014) Interdisziplinarität als Erfolgskonzept beim chronischen Unterbauchschmerzsyndrom. Ergebnisse einer interdisziplinären Spezialsprechstunde für chronische Unterbauchschmerzsyndrome aus psychosomatischem Blickwinkel. Schmerz (im Druck)

3. De Greck M, Andresen V, Layer P (2014) Abdominelle Schmerzen beim Reizdarmsyndrom - ein Überblick über die wichtigsten Empfehlungen. Schmerz (im Druck)

4. Englbrecht JS, Pogatzky-Zahn E (2014) Perioperative Schmerztherapie bei abdominellen und thorakalen Operationen. Schmerz (im Druck)

5. Frettlöh J, Maier C, Gockel H et al 2009 Characterization of chronic pain patients in German pain centers: core data from more than 10,000 patients. Schmerz 23:576-591

6. Görge G, Grandt D, Häuser W (2014) Chronischer Brustschmerz. Schmerz (im Druck)

7. Häuser W, Lempa M, Jänig W (2002) Bauchschmerzen - ein Stiefkind der Schmerztherapie und Forschung? Schmerz 16:425-428

8. Häuser W (2013) Additions required. Dtsch Arztebl Int 110:686
9. Häuser W, Schmutzer G, Brähler E (2014) Häufigkeit von Bauchschmerzen in der allgemeinen Bevölkerung - Befragung einer repräsentativen deutschen Bevölkerungsstichprobe. Schmerz (im Druck)

10. International Association for the Study of Pain (o J) Global year against visceral pain. https://www. iasp-pain.org/Content/NavigationMenu/GlobaIYearAgainstPain/GlobalYearAgainstVisceralPain/ default.htm. Zugegriffen: 27. Oktober 2013

11. Jänig W (Hrsg) (2002) Themenheft Viszeraler Schmerz. Schmerz 16:424-475

12. Jänig W, Baron R (2011) Pathophysiologie des Schmerzes. In: Fischer L, Peuker E (Hrsg) Integrative Schmerztherapie. Carl F. Haug Verlag (MVS Medizinverlage), Stuttgart. S 35-70

13. Jänig W (2014) Neurobiologie viszeraler Schmerzen. Schmerz (im Druck)

14. McMahon SB, Koltzenburg M, Tracey I, Turk DC (Hrsg) (2013) Wall and Melzack's textbook of pain, 6. Aufl. Elsevier Saunders, Philadelphia

15. Moormann O, Gralow I (2014) Chronischer Beckenbodenschmerz. Schmerz (im Druck)

16. Preiß JC, Hoffmann JC (2014) Schmerztherapie bei chronischer Pankreatitis und chronisch-entzündlichen Darmerkrankungen. Schmerz 2014 (im Druck)

17. Schmidt RF, Willis WD (Hrsg) Encyclopedia of pain Springer, Berlin

18. Schwille IJ, Giel KE, Ellert U et al (2009) A community-based survey of abdominal pain prevalence, characteristics, and health care use among children. Clin Gastroenterol Hepatol 7:1062-1068

19. Siedentopf F, Sillem M (2014) Chronischer Unterbauchschmerz der Frau. Schmerz (im Druck)

\section{Chronische Schmerzen: Medikamentöse Langzeit- therapie hat gleichen Effekt wie Placebo}

Starke Schmerzmittel, die über einen längeren Zeitraum gegen chronische Schmerzen eingenommen werden, haben den gleichen Effekt wie eine Behandlung ohne Medikamente. Dies ist das Ergebnis einer umfangreichen Meta-Analyse von Forschern der Charité - Universitätsmedizin Berlin und der Technischen Universität Darmstadt.

Nahezu ein Viertel der gesamten Bevölkerung leitet unter chronischen Schmerzen, die nicht durch einen Tumor hervorgerufen werden. Mit ihrer Studie wollten die Wissenschaftler einen meta-analytischen Überblick zu den Langzeitwirkungen der unterschiedlichen Behandlungsarten chronischer Schmerzen erstellen. Dazu bewerteten sie die Ergebnisse aus insgesamt 3647 Publikationen in internationalen Fachzeitschriften. Von diesen Studien wurden nur die randomisiert-kontrollierten Arbeiten in die weitere Analyse einbezogen. Insgesamt werteten die Forscher die Daten von 10.742 Patienten aus.

Aus der Analyse ging hervor, dass langfristig gesehen die schmerzlindernden Wirkungen von medikamentösen Therapieverfahren klinisch unbedeutend im Vergleich zu einem Placebo sind. Über einen längeren Zeitraum hinweg erziele man mit starken Schmerzmitteln die gleiche Wirkung wie mit nicht medikamentösen physiotherapeutischen und psychologischen Verfahren, erläutern die Autoren.

Literatur: Reinecke H et al (2014) Analgesic efficacy of opioids in chronic pain - recent meta analyses. Br J Pharmacol. doi: 10.1111/bph.12634

Quelle: Charité - Universitätsmedizin Berlin, www.charite.de 